\title{
Destructive Mono-Arthritis Caused by Mycobacterium bovis during Treatment with Pembrolizumab in a Patient Previously Treated with BCG Instillations for Bladder Cancer: A Case Report
}

\author{
${ }^{1}$ Department of Medical Oncology, Erasmus Medical Center, The Netherlands \\ ${ }^{2}$ Department of Respiratory Medicine, Erasmus Medical Center, The Netherlands \\ ${ }^{3}$ Department of Rheumatology, Franciscus Gasthuis \& Vlietland, The Netherlands
}

Cynthia Epskamp ${ }^{1 *}$, Susanne M Huijts ${ }^{2}$, Peter AHM van der Lubbe ${ }^{3}$ and Debbie G.J. Robbrecht ${ }^{1}$

*Corresponding author: Cynthia Epskamp, Department of Medical Oncology, Erasmus MC Cancer Institute, Secretariat IO NT-5, P.O. 2040, 3000 CA, Rotterdam, The Netherlands, Tel: +31107041505 , Fax: +31107041003

\begin{abstract}
Immune checkpoint inhibitor $(\mathrm{ICl})$ treatment is an integral part of second line treatment of patients with urothelial carcinoma (UC) as well as in first line in cisplatin-ineligible patients with PD-L1 positive tumours. A substantial proportion of patients with bladder cancer have been treated with intravesical Mycobacterium bovis BCG in the past. Although it is rare, disseminated infections with Mycobacterium bovis have been described, but not in association with $\mathrm{ICl}$ treatment. We herein report a case of destructive monoarthritis caused by Mycobacterium bovis in a patient previously treated with intravesical BCG and a recent initiation of the $\mathrm{ICI}$ pembrolizumab in first line for metastatic UC. A culture of synovial fluid from the involved wrist was positive for $M y$ cobacterium bovis. Based on whole genome sequencing based-subtyping, the identified Mycobacterium was shown to be related with the previous BCG instillations. Because of severe complaints and progressive destruction of the patients' wrist, it was decided to interrupt the pembrolizumab. At that moment, following the seventh cycle of pembrolizumab, evaluation showed a partial disease response. Following systemic treatment with triple combination therapy (Isoniazid, Rifampicin, Ethambutol) and surgery, the complaints of the wrist gradually improved. This is the first report describing a disseminated Mycobacterium bovis infection in a patient treated with an $\mathrm{ICl}$ and previous BCG instillations for bladder cancer. This is of relevance, because an increasing number of bladder cancer patients, previously treated with BCG instillations, will be treated with an $\mathrm{ICl}$ in advanced disease setting.
\end{abstract}

\section{Keywords}

Immune Checkpoint inhibitor, Bacillus Calmette-Guerin, Mycobacterium bovis, Arthritis, Bladder cancer

\section{List of Abbreviations}

BCG: Bacillus Calmette-Guérin; CIS: Carcinoma in situ; ICI: Immune checkpoint inhibitor; CTCAE: Common Terminology Criteria for Adverse Events; PD-1: Programmed death-1; SNPs: Single nucleotide polymorphisms; TBC: Tuberculosis; TNF: Tumor necrosis factor; TURB: Transurethral resection of the bladder; UC: Urothelial carcinoma

\section{Background}

Patients with non-muscle invasive urothelial cancer can be treated with intravesical administration of Bacillus Calmette-Guérin (BCG), a live attenuated strain of Mycobacterium bovis (M. bovis). In general, this treatment is well tolerated, although disseminated BCG infection can occur. This is uncommon but a well-recognized complication of intravesical BCG treatment [1-4]. The underlying mechanism by which intravesical BCG leads to systemic infections is not fully understood. The rate of osteoarticular side effects after intravesical BCG therapy ranges from 0.5 to 1 percent [5].

Despite treatment with intravesical BCG and TURB, approximately $40 \%$ of the patients progress to muscle invasive disease and progression to metastatic urothelial cancer occurs in 20 to $30 \%$ of these patients $[6,7]$. In patients with metastatic urothelial cell carcinoma with progressive disease following or during first line platinum based chemotherapy, the FDA approved treatment

Citation: Epskamp C, Huijts SM, Lubbe PAHM, Robbrecht DGJ (2021) Destructive Mono-Arthritis Caused by Mycobacterium bovis during Treatment with Pembrolizumab in a Patient Previously Treated with BCG Instillations for Bladder Cancer: A Case Report. Int J Oncol Res 4:026. doi. org/10.23937/2643-4563/1710026

Accepted: February 08, 2021: Published: February 10, 2021

Copyright: (c) 2021 Epskamp C, et al. This is an open-access article distributed under the terms of the Creative Commons Attribution License, which permits unrestricted use, distribution, and reproduction in any medium, provided the original author and source are credited. 
Table 1: Differences in SNPs (number) based on WGS.

\begin{tabular}{|c|c|c|c|c|c|}
\hline & NLA001900744 & $\begin{array}{l}\text { M. bovis BCG } \\
\text { vaccin strain 1 }\end{array}$ & $\begin{array}{l}\text { M. bovis BCG } \\
\text { vaccin strain 2 }\end{array}$ & $\begin{array}{l}\text { M. bovis BCG } \\
\text { installation strain }\end{array}$ & $\begin{array}{l}\text { WGS fingerprint } \\
\text { type }\end{array}$ \\
\hline NLA001900744 & - & & & & $A 003$ \\
\hline A004 & 27 & - & & $A 004$ \\
\hline A005 & 61 & 46 & - & $A 005$ \\
\hline A003 & 5 & 24 & 58 & - & $A 003$ \\
\hline
\end{tabular}

A003: $M$. bovis BCG installation strain; A004: M. bovis BCG vaccin strain 1; A005: M. bovis BCG vaccin strain 2; NLA001900744: $M$. bovis strain of the patient

with an immune checkpoint inhibitor (ICl) targeting PD1 or PD-L1 (pembrolizumab, atezolizumab, durvalumab, avelumab, nivolumab) as second line treatment [8-12]. There have been only a few reports of a flare of tuberculosis in patients treated with immune checkpoint inhibitors (ICls), but no reports of $M$. bovis related infections [13-17].

Here, we present a patient previously treated with intravesical BCG who developed a fulminant $M$. bovis related mono-arthritis during treatment with pembrolizumab for advanced bladder cancer.

\section{Case Presentation}

Our 64-year-old Caucasian male patient was diagnosed in January 2017 with a non-invasive papillary carcinoma of the urinary bladder (cTaG3). He was treated with 6 cycles of intravesical BCG. In May 2017 a re-transurethral resection of the bladder (TURB) showed a T1G3 urothelial cell carcinoma for which he received re-induction with 6 cycles of intravesical BCG. Unfortunately, the patient developed metastatic disease in June 2019. As a results of preexisting grade 2 tinnitus the patient was deemed to be cisplatinum ineligible and therefore PD-L1 expression was assessed by immunohistochemistry on tissue derived from the earlier TURB by using the Dako PD-L1 IHC 22C3 test. Based on a combined positive score (CPS) result of $>10$, the patient was candidate to be treated with pembrolizumab. In July 2019, treatment with pembrolizumab $200 \mathrm{mg}$ ( $\mathrm{q} 3 \mathrm{w}$ ) was initiated. Response evaluation following cycle 4 showed a partial response based on RECIST 1.1. [18]. Adverse events were fatigue grade 1 and a rash grade 1 according to the Common Terminology Criteria for Adverse Events (CTCAE) Version 4.0 [19]. The patient reported also increasing pain and impaired movement of his left wrist.

Six months before the initiation of the pembrolizum$a b$ the patient presented at the outpatient rheumatology clinic with a slight synovial swelling of the left wrist without impairment of the joint. Retrospectively the first complaints of the wrist started some 18 months before and gradually evolved over time. Analysis revealed a markedly positive IgM-rheumatoid factor and a negative anti-CCP and no abnormalities were seen with plain radiography of the wrist. Treatment with a non-steroidal anti-inflammatory drug (ibuprofen) was initiated. Because of an insufficient effect, the patient was subsequently treated with a triamcinolone injection of the wrist. The working diagnosis of his rheumatologist was a monoarthritis either as a first sign of a starting rheumatoid arthritis or otherwise a paraneoplastic syndrome. Awaiting further diagnostic procedures and the subsequent decision concerning the treatment of the patients' advanced urothelial carcinoma, it was decided not to start disease modifying ant rheumatic drugs but to continue with local intra-articular triamcinolone injection when indicated. Two weeks following the first cycle of pembrolizumab, the patient developed a clear increase of complaints of his wrist for which he received an intra-articular triamcinolone injection again. This time without significant effect. Plain radiography of the wrist showed an increasing osteopenia. An MRI scan and a diagnostic puncture with aspiration of the synovial fluid of the left wrist were performed. The MRI scan showed a very active synovial inflammation and serious erosive changes of the intercarpal and carpometocarpal joints. The culture of the synovial fluid turned out to be positive with $M$. bovis. The identified $M$. bovis strain was compared with the $M$. bovis strain used in BCG instillations, by using whole genome sequencing (Table 1 ). In this analysis WGS patterns were compared between 2 available BCG vaccination strains, 1 BCG instillation strain and the strain of our patient. The latter and the BCG instillation strain turned out to differ only 5 single nucleotide polymorphisms (SNPs). To show a correlation, strains should differ less than 12 SNPs. Based on the performed analysis, it can be concluded that the arthritis in our patient was caused by an M. bovis infection as a consequence of his previous treatment with BCG instillations.

Triple combination treatment was started with Isoniazid, Rifampicin and Ethambutol, with discontinuation of Ethambutol after one month based on the resistance pattern of the identified M. bovis. Unfortunately, two months later the complaints aggravated caused by an ongoing bacterial arthritis with extension to the ulnocarpal, intercarpal and carpometocarpal joints with secondary abscess formation for which the patient underwent surgical debridement and synoviectomy. After recovery from surgery, the patient remained free of complaints. Isoniazid and Rifampicin were discontinued 9 months after initiation. The pembrolizumab was kept interrupted and 8 months later there still is an ongoing partial response of this treatment. 


\section{Discussion and Conclusions}

Serious systemic side effects of treatment with intravesical BCG installations are rare, with an incidence of pneumonitis or hepatitis of $0.7 \%$ and osteoarticular side effects of $0.5 \%[20,21]$. Although one study reported an incidence of $4.3 \%$ of systemic BCG infection [22], disseminated BCG-induced infections can manifest many years later [21]. It has been reported that BCG can persist in the bladder for more than one year after intravesical BCG installation [20]. Osteoarticular side effects are mostly manifested by reactive arthritis, but an infectious arthritis by $M$. bovis can also occur, predominantly in the lower extremities [22-26].

The mechanism of action of BCG-induced systemic infections is unclear. Even the exact working mechanism of treatment with intravesical of BCG is unknown. It is assumed that BCG installation triggers a variety of local immune responses, which includes the following: Induction of mononuclear cell infiltrate, consisting of CD4 T cells and macrophages; increased expression of interferon gamma) in the bladder; direct suppression of tumor growth; elevating urinary cytokine levels including interleukin-1, $-2,-8$ and 12 , interferon-gamma, tumor necrosis factor (TNF) alpha and tumor necrosis factor apoptosis inducing ligand [27]. In synovial fluid and tissue in patients with BCG arthritis antigen-specific CD4+ and CD8+ T cells have been found [24].

To the best of our knowledge reactivation of latent $M$. bovis infection has not been described in patients treated with an $\mathrm{ICl}$. Aggravation of latent mycobacterium infections during treatment with an $\mathrm{ICl}$ is a concern of clinicians. It has been described that reactivation of latent $M$. tuberculosis (TBC) infections in patients treated with PD-1 targeted therapy is possible. One article summarized 11 case reports documenting patients treated with PD-1 inhibitors developing acute pulmonary tuberculosis [16]. In our patient there were no signs of pulmonary involvement. Patients with latent TBC seem to develop reactivation and symptoms within 6 months of PD-1/PD-L1 inhibition [15]. This was also the case in our patient. A retrospective analysis showed an association between the use of PD-1/PD-L1 inhibitors and increased TBC and atypical mycobacterial infection, with a reporting odds ratio of 1.79 and 5.49 respectively [17].

There are hypotheses about potential mechanisms of TBC reactivation caused by immune checkpoint inhibition. It has been hypothesized that progression from latent TBC to an active infection is a consequence of failure of the immune system, as demonstrated by increased incidence in patients with an HIV infection or after anti-tumor necrosis factor treatment. PD-1 inhibition has been suggested as a host-directed therapy in TBC, on the basis that the PD-1 pathway may inhibit an effective host response [14]. In mouse models enhanced CD4 activity in absence of PD-1 surveillance exacerbates tuberculosis. In human patients with active TBC, PD-1 was described to be increased on CD4+ T cells but not on CD8+ T cells compared to healthy controls, while effective anti-tuberculosis treatment was associated with downregulation of PD-1 on CD4+ T cells $[28,29]$. One study suggests TNF-alpha may be the driver of TBC reactivation associated with PD-1 inhibition [30].

In conclusion, we present a patient with a proven BCG-instillation related $M$. bovis mono-arthritis in whom a relatively indolent inflammation changed into a fulminant and severe arthritis during treatment with an immune checkpoint inhibitor. The aggravation of the inflammation could be a result of the $\mathrm{ICl}$ used. To our knowledge this is the first case in literature. Clinicians should be aware of this complication since an increasing number of patients will be treated with $\mathrm{ICl}$ in advanced urothelial cancer setting after previous treatment with BCG instillations.

\section{Compliance with Ethical Standards}

\section{Conflict of interest}

The authors declare that they have no conflict of interest.

\section{Informed consent}

Written informed consent was obtained from the patient for publication of this case report.

\section{Declarations}

\section{Funding}

Not applicable.

\section{Availability of date and material}

Not applicable.

\section{Code availability}

Not applicable.

\section{Authors' Contributions}

$\mathrm{DR}, \mathrm{SH}$ and PL treated the patient. CE and DR wrote the case report. All authors made substantial contributions to discussion, review and revision. All authors read and approved the final manuscript.

\section{References}

1. Lotte A, Wasz-Höckert O, Poisson N, Dumitrescu N, M Verron, et al. (1984) BCG complication. Estimates of the risks among vaccinated subjects and statistical analysis of their main characteristics. Adv Tuberc Res 21: 107-193.

2. Rosenberg EB, Kanner SP, Schwartzman RJ (1974) Systemic infection following BCG therapy. Arch Intern Med 134: 769-770.

3. Talbot EA, Perkins MD, Silva SF, Frothingham R (1997) Disseminated bacille Calmette-Guerin disease after vaccination: case report and review. Clin Infect Dis 24: 11391146.

4. Aljada IS, Crane JK, Corriere N, Wagle DG, Amsterdam D (1999) Mycobacterium bovis BCG causing vertebral osteo- 
myelitis (Pott's disease) following intravesical BCG therapy. J Clin Microbiol 37: 2106-2108.

5. Lamm DL, Stogdill VD, Stogdill BK, Crispen RG (1986) Complications of bacillus Calmette-Guerin immunotherapy in 1,278 patients with bladder cancer. J Urol 135: 272-274.

6. Cookson MS, Herr HW, Zhang ZF, Soloway S, Sogani PC, et al. (1997) The treated natural history of high risk superficial bladder cancer: 15-year outcome. J Urol 158: 62-67.

7. Millán-Rodríguez F, Chéchile-Toniolo G, Salvador-Bayarri J, Palou J, Algabaj F, et al. (2000) Primary superficial bladder cancer risk groups according to progression, mortality and recurrence. J Urol 164: 680-684.

8. Bellmunt $\mathrm{J}$, de Wit $\mathrm{R}$, Vaughin $\mathrm{DJ}$, Fradet $\mathrm{Y}$, Lee $\mathrm{JL}$, et al. (2017) KEYNOTE-045 Investigators. Pembrolizumab as second-line therapy for advanced urothelial carcinoma. $\mathrm{N}$ Engl J Med 376: 1015-1026.

9. Rosenberg JE, Hoffman-Censits J, Powles T, van der Heijden MS, Balar AV, et al. (2016) Atezolizumab in patients with locally advanced and metastatic urothelial carcinoma who have progressed following treatment with platinum-based chemotherapy: a single-arm, multicentre, phase 2 trial. Lancet 387: 1909-1920.

10. Powles T, O'Donnel PH, Massard C, Arkenau HT, Friedlander TW, et al. (2017) Efficacy and safety of durvalumab in locally advanced or metastatic urothelial carcinoma Updated results from a phase 1/2 open-label study. JAMA Oncol 3: e172411.

11. Patel MR, Ellerton J, Infante JR, Agrawal M, Gordon M, et al. (2018) Avelumab in metastatic urothelial carcinoma after platinum failure (JAVELIN Solid Tumor): pooledresults from two expansion cohorts of an open-label, phase 1 trial. Lancet Oncol 19: 51-64.

12. Sharma $P$, Retz $M$, Siefker-Radtke A, Baron A, Necchi $A$, et al. (2017) Nivolumab in metastatic urothelial carcinoma after platinum therapy (CheckMate 275): a multicentre, single-arm, phase 2 trial. Lancet Oncol 18: 312-322.

13. Tezera LB, Mansour S, Elkington P (2020) Reconsidering the optimal immune response to mycobacterium tuberculosis. Am J Respir Crit Care Med 201: 407-413.

14. Elkington PT, Bateman AC, Thomas GJ, Ottensmeier $\mathrm{CH}$ (2018) Implications of tuberculosis reactivation after immune checkpoint inhibition. Am J Respir Crit Care Med 198: 1451-1453.

15. Anastasopoulou A, Ziogas DC, Samarkos M, Kirkwood JM, Gogas H (2019) Reactivation of tuberculosis in cancer patients following administration of immune checkpoint inhibitors: current evidence and clinical practice recommendations. J Immunother Cancer 7: 239.

16. van Eeden R, Rapoport B, Smit T, Anderson R (2019) Tuberculosis infection in a patient treated with nivolumab for non-small cell lung cancer: case report and literature review. Frontiers in oncology 9: 1-5.
17. Anand K, Sahu G, Burns E, Ensor A, Ensor J, et al. (2020) Mycobacterial infections due to PD-1 and PD-L1 checkpoint inhibitors. ESMO Open 5: e000866.

18. Eisenhower EA, Therasse P, Bogaerts J, Sargent D, Ford $R$, et al. (2009) New response evaluation criteria in solid tumours: revised RECIST guideline (version 1.1). Eur $\mathrm{J}$ Cancer 45: 228-247.

19. U.S. Department of health and human services (2009) Common Terminology Criteria for Adverse Events (CTCAE) Version 4.02010.

20. Lamm DL, Steg A, Boccon-Gibod L, Morales A, Hanna Jr MG, et al. (1989) Complications of Bacillus Calmette-Guerin immunotherapy: review of 2602 patients and comparison of chemotherapy complications. Prog Clin Biol Res 310: 335-355.

21. Steg A, Adjiman S, Debré S (1992) BCG therapy in superficial bladder tumours-complications and precautions. Eur Urol 21: 35-40.

22. Pérez-Jacoiste Asín MA, Fernández-Ruiz M, López-Medrano F, Lumbreras C, Tejido A, et al. (2014) Bacillus CalmetteGuérin (BCG) infection following intravesical BCG administration as adjunctive therapy for bladder cancer: incidence, risk factors, and outcome in a single-institution series and review of the literature. Medicine 93: 236-254.

23. Clavel G, Grados F, Lefauveau P, Fardellone P (2006) Osteoarticular side effects of BCG therapy. Joint Bone Spine 73: 24-28.

24. Tinazzi E, Ficarri V, Simeoni S, Artibani W, Lunardi C (2006) Reactive arthritis following BCG immunotherapy for urinary bladder carcinoma: a systematic review. Rheumat Int 26: 481-488.

25. Miranda S, Verdet M, Heron F, Vittecoq O, Levesque $\mathrm{H}$, et al. (2010) Acute reactive arthritis after intravesical installation of bacillus Calmette-Guerin. Two case reports and literature review. Rev Med Intern 31: 558.

26. Bernini L, Umberto Manzini C, Giuggioli D, Sebastiani M, Ferri C (2013) Reactive arthritis induced by intravesical BCG therapy for bladder cancer: our clinical experience and systematic review of the literature. Autoimmunity $\mathrm{Re}-$ views 12: 1150-1159.

27. O'Donnel MA, Orr PH (2020) Infectious complications of intravesical BCG immunotherapy.

28. Shen L, Gao Y, Liu Y, Zhang B, Liu Q, et al. (2016) PD-1/ PD-L pathway inhibits Mtb-specific CD4(+) T-cell functions and phagocytosis of macrophages in active tuberculosis. Sci Rep 6: 38362.

29. Shen L, Shi H, Gao Y, Ou Q, Liu Q, et al. (2016) The characteristic profiles of PD-1 and PD-L1 expressions and dynamic changes during treatment in active tuberculosis. Tuberculosis (Edinb) 101: 146-150.

30. Tezera LB, Bielecka MK, Ongongo P, Walker NF, Ellis M, et al. (2020) Anti-PD-1 immunotherapy leads to tuberculosis reactivation via dysregulation of TNF-a. Elife 9: e52668.. 